\title{
On Peculiarities of Propagation of a Plane Elastic Wave through a Gradient Anisotropic Layer
}

\section{Anastasiia Anufrieva, Dmitry Chickrin, and Dmitrii Tumakov}

Kazan Federal University, 18 Kremlyovskaya Street, Kazan 420008, Russia

Correspondence should be addressed to Dmitrii Tumakov; dtumakov@kpfu.ru

Received 15 October 2015; Accepted 3 December 2015

Academic Editor: Kim M. Liew

Copyright (c) 2015 Anastasiia Anufrieva et al. This is an open access article distributed under the Creative Commons Attribution License, which permits unrestricted use, distribution, and reproduction in any medium, provided the original work is properly cited.

The problem of diffraction of a plane elastic wave by an anisotropic layer is studied. The diffraction problem is reduced to a boundary value problem for the layer. The grid method is used for solving the resulting boundary value problem. The diffraction of a plane longitudinal wave by the layer is considered. Some peculiarities of the gain-frequency and the gain-angle characteristics of a normal component of an energy flow of a passed longitudinal wave are numerically studied.

\section{Introduction}

Compared to homogenous and isotropic materials, anisotropic materials represent an enormous interest from the practical point of view. Their properties vary in different spatial directions. Anisotropic materials and media are encountered in nature and they are often used in various industries.

During the last 50 years, interest to anisotropic materials has become strong, especially from research groups working in the areas of geology [1-4] and seismology [5-7]. It is well recognized that anisotropy of geological formations is caused by stratification of the prevalent direction of microcracks as well as by orientation of elements of geological formations possessing initial anisotropy. It is worth noting that this type of anisotropy originates from crystal texture of the materials. Experimental investigations [8] indicate that the anisotropy is caused by stresses induced by the rock masses. All of this allowed geologists to gain a better understanding of structure of the Earth's crust [9] and motivated further studies in the area of anisotropic materials.

One more research direction of the area of anisotropic materials is acoustic metamaterials and phononic crystals [10]. These materials are artificial composite materials possessing a rather complicated structure [11, 12]. Investigation of these kinds of materials allowed making a huge progress in such engineering areas as developing good screening, noise insulation, and reaching full control over the ratio of energy of the transmitted wave to that of the reflected wave [13].

It is recognized that elastic properties of materials can often vary in the point-to-point manner. Sometimes, variation of elastic properties of geological layers with the depth can be caused by stresses in the surrounding rock masses. In industrial production of layered structures, one can create continuous-type variations of the ratios of the included constituent matters and, thereby, obtain a sufficiently smooth variation of the resulting material properties. By doing this way, various materials possessing a continuous (gradient) distribution of elastic characteristics in the transverse direction and homogeneous characteristics in the longitudinal direction of the layer can be produced.

Aside from variation of elastic properties in the point-topoint manner, another type of variation across the material is also quite often encountered both in nature and in industries. The variation is in the abrupt changing of the material's elastic properties from one layer to another. One of the approaches to modeling such layered structures is associated with applying of a stratified model to the anisotropic material [14].

One of the first and simplest approaches to solving the problem was related to analysis of diffraction of elastic waves 
by isotropic layers possessing a continuous distribution of parameters. However, it is noteworthy that a more precise approach to an anisotropic model is in treating it as a transversely isotropic model. This approach was applied by our research group in the past to investigate the problem of elastic wave propagation through gradient isotropic materials [15] and gradient transversely isotropic materials [16, 17]. In [18], diffraction of plane acoustic wave by inhomogeneous layer was investigated. Sonic wave transmission through inhomogeneous anisotropic layer adjacent to a viscous fluid was investigated in [19].

In the present work, diffraction of elastic waves by an inhomogeneous anisotropic layer is chosen to be a subject of our investigation. Differential equations governing the diffraction problem for half-planes are considered separately from those for the layer. The problems for half-planes are overdetermined (using the overdetermination is, indeed, the essence of our proprietary approach) allowing establishing a relationship between traces of desired functions at the media's interface [20]. Thus, the original problem reduces to the boundary value problem for the partial differential equations system with boundary conditions of the third type. After that, we applied the Fourier transform with respect to the variable, for which uniformity is preserved, to the resulting boundary value problem. The obtained system of ordinary differential equations was solved using the grid method.

Let us note that the algorithm was applied by our research group in the past not only in the works cited above but also in a number of other works of our group as well. For example, the problem of elastic wave diffraction by the layer with the fractal distribution of density was considered in [21].

Numerical results of the elastic wave propagation through a gradient layer with a linear variation of parameters, which are similar to properties of sandstone, are presented in the present work. Peculiarities of the wave propagation through such layers are shown. The transmission range for the gradient anisotropic layer at angle values close to the tangent angle is given. In addition, an example of passage of an elastic wave through a layer having parameters varying in the continuous manner from amazonite to labradorite is considered.

\section{Statement of the Problem}

Let an elastic harmonic wave of type $\mathbf{u}_{0}(x, y) \exp \{i \omega t\}$ fall on anisotropic layer of thickness $L$ (medium $2\{0<y<L\}$ ), which is a nonuniform in the transverse direction, with continuous density $\rho_{2}(y)$ and tensor of elasticity modulus $\mathbf{K}(y)$ from medium $1\{y>L\}$ under the angle $\theta$ with respect to the axis $y$ (see Figure 1). The diffraction result is a wave $\mathbf{u}_{1}(x, y)$ reflected toward medium 1 and a wave $\mathbf{u}_{3}(x, y)$ propagating toward medium $3\{y<0\}$ and field $\mathbf{u}_{\mathbf{2}}(x, y)$ in the layer. We need to investigate response frequency characteristics of the longitudinal part of the wave $\mathbf{u}_{3}(x, y)$. Media 1 and 3 are assumed to be uniform and isotropic. Medium 2 is assumed to be of the gradient type and anisotropic.

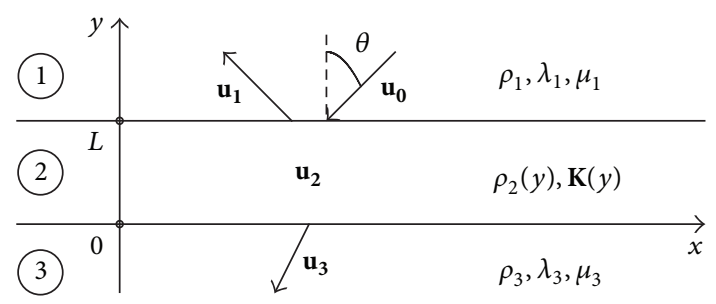

Figure 1: Geometry of the problem.

We seek a solution to the plane harmonic problem from the elasticity theory at $y<0$ and $y>L$ in the following form:

$$
\begin{aligned}
\frac{\partial \sigma_{x n}}{\partial x}+\frac{\partial \tau_{n}}{\partial y}+\rho_{n} \omega^{2} u_{x n} & =0 \\
\frac{\partial \tau_{n}}{\partial x}+\frac{\partial \sigma_{y n}}{\partial y}+\rho_{n} \omega^{2} u_{y n} & =0 \\
\sigma_{x n} & =\left(\lambda_{n}+2 \mu_{n}\right) \frac{\partial u_{x n}}{\partial x}+\lambda_{n} \frac{\partial u_{y n}}{\partial y}, \\
\sigma_{y n} & =\lambda_{n} \frac{\partial u_{x n}}{\partial x}+\left(\lambda_{n}+2 \mu_{n}\right) \frac{\partial u_{y n}}{\partial y}, \\
\tau_{n} & =\mu_{n}\left(\frac{\partial u_{x n}}{\partial y}+\frac{\partial u_{y n}}{\partial x}\right)
\end{aligned}
$$

for $n=1,3$ with the constant Lame coefficients $\lambda_{n}, \mu_{n}$ and density $\rho_{n}$.

Equations of two-dimensional oscillations in anisotropic media are written in the following form:

$$
\begin{aligned}
-\rho_{2} \omega^{2} u_{x 2}= & \frac{\partial \sigma_{x 2}}{\partial x}+\frac{\partial \tau_{2}}{\partial y}, \\
-\rho_{2} \omega^{2} u_{y 2}= & \frac{\partial \tau_{2}}{\partial x}+\frac{\partial \sigma_{y 2}}{\partial y}, \\
\sigma_{x 2}= & k_{x x x x} \frac{\partial u_{x 2}}{\partial x}+k_{x x x y}\left(\frac{\partial u_{x 2}}{\partial y}+\frac{\partial u_{y 2}}{\partial x}\right) \\
& +k_{x x y y} \frac{\partial u_{y 2}}{\partial y}, \\
\sigma_{y 2}= & k_{y y x x} \frac{\partial u_{x 2}}{\partial x}+k_{y y x y}\left(\frac{\partial u_{x 2}}{\partial y}+\frac{\partial u_{y 2}}{\partial x}\right) \\
& +k_{y y y y} \frac{\partial u_{y 2}}{\partial y}, \\
\tau_{2}= & k_{x y x x} \frac{\partial u_{x 2}}{\partial x}+k_{x y x y}\left(\frac{\partial u_{x 2}}{\partial y}+\frac{\partial u_{y 2}}{\partial x}\right) \\
& +k_{x y y y} \frac{\partial u_{y 2}}{\partial y},
\end{aligned}
$$

where $k_{* * * *}$ are components of the elasticity modulus tensor $\mathbf{K}$. Let us introduce a standard denotation for indices 
$k_{* * * *}:\{x x\} \rightarrow\{1\},\{y y\} \rightarrow\{2\}$, and $\{x y\} \rightarrow\{3\}$. Substitution of (3) into (2) gives

$$
\begin{aligned}
& -\rho_{2} \omega^{2} u_{x 2}=\frac{\partial}{\partial x}\left(k_{11} \frac{\partial u_{x 2}}{\partial x}+k_{13}\left(\frac{\partial u_{x 2}}{\partial y}+\frac{\partial u_{y 2}}{\partial x}\right)\right. \\
& \left.+k_{12} \frac{\partial u_{y 2}}{\partial y}\right)+\frac{\partial}{\partial y}\left(k_{13} \frac{\partial u_{x 2}}{\partial x}+k_{33}\left(\frac{\partial u_{x 2}}{\partial y}+\frac{\partial u_{y 2}}{\partial x}\right)\right. \\
& \left.+k_{23} \frac{\partial u_{y 2}}{\partial y}\right), \\
& -\rho_{2} \omega^{2} u_{y 2}=\frac{\partial}{\partial x} k_{13} \frac{\partial u_{x 2}}{\partial x}+k_{33}\left(\frac{\partial u_{x 2}}{\partial y}+\frac{\partial u_{y 2}}{\partial x}\right)+k_{23} \\
& +\frac{\partial u_{y 2}}{\partial y}+\frac{\partial}{\partial y}\left(k_{12} \frac{\partial u_{x 2}}{\partial x}+k_{23}\left(\frac{\partial u_{x 2}}{\partial y}+\frac{\partial u_{y 2}}{\partial x}\right)\right. \\
& \left.+k_{22} \frac{\partial u_{y 2}}{\partial y}\right) .
\end{aligned}
$$

At the media's interface, the following conjugation conditions are to be fulfilled:

$$
\begin{gathered}
u_{x 1}(x, L+0)+u_{x 0}(x, L+0)=u_{x 2}(x, L-0), \\
u_{y 1}(x, L+0)+u_{y 0}(x, L+0)=u_{y 2}(x, L-0), \\
\tau_{1}(x, L+0)+\tau_{0}(x, L+0)=\tau_{2}(x, L-0), \\
\sigma_{y 1}(x, L+0)+\sigma_{y 0}(x, L+0)=\sigma_{y 2}(x, L-0)
\end{gathered}
$$

at $y=L$ and

$$
\begin{gathered}
u_{x 3}(x, 0-0)=u_{x 2}(x, 0+0), \\
u_{y 3}(x, 0-0)=u_{y 2}(x, 0+0), \\
\tau_{3}(x, 0-0)=\tau_{2}(x, 0+0), \\
\sigma_{y 3}(x, 0-0)=\sigma_{y 2}(x, 0+0)
\end{gathered}
$$

at $y=0$.

Of all the possible solutions to system (1) and (4) with the conjugation conditions, we pick solutions corresponding to the waves going to infinity.

\section{Solution of the Diffraction Problem}

We apply the Fourier transform to (4) with respect to the $x$-direction permitting both waves decaying at infinity and propagating waves. Thus, we perform the change of variables from variable $x$ to variable $\xi$ and obtain the system of equations at $y \in(0, L)$ :

$$
\begin{aligned}
& k_{33} u_{x 2}^{\prime \prime}+\left[k_{33}^{\prime}-2 i \xi k_{13}\right] u_{x 2}^{\prime} \\
& +\left[\rho_{2} \omega^{2}-k_{11} \xi^{2}-i \xi k_{13}^{\prime}\right] u_{x 2}+k_{23} u_{y 2}^{\prime \prime} \\
& +\left[k_{23}^{\prime}-i \xi\left(k_{12}+k_{33}\right)\right] u_{y 2}^{\prime} \\
& +\xi\left[i k_{33}^{\prime}+\xi k_{13}\right] u_{y 2}=0, \\
& k_{22} u_{y 2}^{\prime \prime}+\left[k_{22}^{\prime}-2 i \xi k_{23}\right] u_{y 2}^{\prime} \\
& +\left[\rho_{2} \omega^{2}-k_{33} \xi^{2}-i \xi k_{23}^{\prime}\right] u_{y 2}+k_{23} u_{x 2}^{\prime \prime} \\
& +\left[k_{23}^{\prime}-i \xi\left(k_{12}+k_{33}\right)\right] u_{x 2}^{\prime} \\
& -\xi\left[i k_{12}^{\prime}+\xi k_{13}\right] u_{x 2}=0,
\end{aligned}
$$

with respect to the Fourier transform for displacements $u_{x 2}(\xi, y)$ and $u_{y 2}(\xi, y)$.

Next, we obtain equations linking traces of Fourier transforms of displacements in the layer with each other at the upper boundary [16]:

$$
\begin{aligned}
& a_{1}(\xi) u_{x 2}^{\prime}(\xi, L)+a_{2}(\xi) u_{x 2}(\xi, L)+a_{3}(\xi) u_{y 2}^{\prime}(\xi, L) \\
& \quad+a_{4}(\xi) u_{y 2}(\xi, L)=f_{1}(\xi), \\
& a_{5}(\xi) u_{x 2}^{\prime}(\xi, L)+a_{6}(\xi) u_{x 2}(\xi, L)+a_{7}(\xi) u_{y 2}^{\prime}(\xi, L) \\
& \quad+a_{8}(\xi) u_{y 2}(\xi, L)=f_{2}(\xi),
\end{aligned}
$$

where

$$
\begin{aligned}
& a_{1}(\xi)=k_{33}(L), \\
& a_{2}(\xi)=-\left(k_{12}(L)+2 \mu_{1}\right) i \xi \gamma_{1,1}(\xi), \\
& a_{3}(\xi)=k_{22}(L) \gamma_{1,1}(\xi), \\
& a_{4}(\xi)=-i\left(\rho_{1} \omega^{2}-\left(2 \mu_{1}-k_{33}(L)\right) \xi^{2}\right), \\
& a_{5}(\xi)=-k_{33}(L) \gamma_{2,1}(\xi), \\
& a_{6}(\xi)=i\left(\rho_{1} \omega^{2}-\left(k_{12}(L)+2 \mu_{1}\right) \xi^{2}\right), \\
& a_{7}(\xi)=k_{22}(L) \xi, \\
& a_{8}(\xi)=-i\left(2 \mu_{1}-k_{33}(L)\right) \xi \gamma_{2,1}(\xi),
\end{aligned}
$$

and right-hand sides are as follows:

$$
\begin{aligned}
f_{1}(\xi)= & \xi \tau_{0}(\xi, L)+\gamma_{1,1}(\xi) \sigma_{y 0}(\xi, L) \\
& -2 i \mu_{1} \xi \gamma_{1,1}(\xi) u_{x 0}(\xi, L) \\
& -i\left(\rho_{1} \omega^{2}-2 \mu_{1} \xi^{2}\right) u_{y 0}(\xi, L)
\end{aligned}
$$




$$
\begin{aligned}
f_{2}(\xi)= & -\gamma_{2,1}(\xi) \tau_{0}(\xi, L)+\xi \sigma_{y 0}(\xi, L) \\
& +i\left(\rho_{1} \omega^{2}-2 \mu_{1} \xi^{2}\right) u_{x 0}(\xi, L) \\
& -2 i \mu_{1} \xi \gamma_{2,1}(\xi) u_{y 0}(\xi, L) .
\end{aligned}
$$

Here $k_{11}^{2}=\rho_{1} \omega^{2} /\left(\lambda_{1}+2 \mu_{1}\right), k_{21}^{2}=\rho_{1} \omega^{2} / \mu_{1}$, and branches of roots of the functions $\gamma_{1,1}=\sqrt{k_{11}^{2}-\xi^{2}}, \gamma_{2,1}=\sqrt{k_{21}^{2}-\xi^{2}}$ are chosen such that the real part is positive, and in the case of the real part being zero, positive imaginary roots are chosen.

For the lower boundary conditions, we obtain [16]

$$
\begin{aligned}
& b_{1}(\xi) u_{x 2}^{\prime}(\xi, 0)+b_{2}(\xi) u_{x 2}(\xi, 0)+b_{3}(\xi) u_{y 2}^{\prime}(\xi, 0) \\
& \quad+b_{4}(\xi) u_{y 2}(\xi, 0)=0, \\
& b_{5}(\xi) u_{x 2}^{\prime}(\xi, 0)+b_{6}(\xi) u_{x 2}(\xi, 0)+b_{7}(\xi) u_{y 2}^{\prime}(\xi, 0) \\
& \quad+b_{8}(\xi) u_{y 2}(\xi, 0)=0,
\end{aligned}
$$

where

$$
\begin{aligned}
& b_{1}(\xi)=k_{33}(0) \xi \\
& b_{2}(\xi)=i\left(k_{12}(0)+2 \mu_{3}\right) \xi \gamma_{1,3}(\xi), \\
& b_{3}(\xi)=-k_{22}(0) \gamma_{1,3}(\xi), \\
& b_{4}(\xi)=-i\left(\rho_{3} \omega^{2}-\left(2 \mu_{3}-k_{33}(0)\right) \xi^{2}\right), \\
& b_{5}(\xi)=k_{33}(0) \gamma_{2,3}(\xi) \\
& b_{6}(\xi)=i\left(\rho_{3} \omega^{2}-\left(k_{12}(0)+2 \mu_{3}\right) \xi^{2}\right), \\
& b_{7}(\xi)=k_{22}(0) \xi \\
& b_{8}(\xi)=i\left(2 \mu_{3}-k_{33}(0)\right) \xi \gamma_{2,3}(\xi) .
\end{aligned}
$$

Here $k_{13}^{2}=\rho_{3} \omega^{2} /\left(\lambda_{3}+2 \mu_{3}\right), k_{23}^{2}=\rho_{3} \omega^{2} / \mu_{3}$, and branches of roots of the functions $\gamma_{1,3}=\sqrt{k_{13}^{2}-\xi^{2}}, \gamma_{2,3}=\sqrt{k_{23}^{2}-\xi^{2}}$ are chosen in the same way as branches of roots of the functions of the upper half-plane.

Physical meanings of solutions of (7) with boundary conditions (8) and (11) are displacements $\left(u_{x 2}, u_{y 2}\right)$, which describe the field at $0<y<L$ in the problem of diffraction by the elastic gradient anisotropic layer.

\section{Numerical Results}

Let us conduct numerical experiments for several synthetic materials. Material 1 is made as the gradient-type anisotropic structure with continuous distribution of tensor elasticity elements in accordance with the law: first values of elasticity
TABLE 1: Zero and nonzero elements $k_{i j}$ for materials 1-4.

\begin{tabular}{ccccccc}
\hline Material 1 & $k_{11}$ & $k_{12}$ & $k_{13}$ & $k_{22}$ & $k_{23}$ & $k_{33}$ \\
\hline Material 2 & $k_{11}$ & $k_{12}$ & 0 & $k_{22}$ & $k_{23}$ & $k_{33}$ \\
\hline Material 3 & $k_{11}$ & $k_{12}$ & $k_{13}$ & $k_{22}$ & 0 & $k_{33}$ \\
\hline Material 4 & $k_{11}$ & $k_{12}$ & 0 & $k_{22}$ & 0 & $k_{33}$ \\
\hline
\end{tabular}

tensor linearly increase from values of elements of elasticity tensor $\mathbf{K}_{\mathbf{1}}$ to values of elements of $\mathbf{K}_{2}$ :

$$
\begin{aligned}
& \mathbf{K}_{1}=\left(\begin{array}{lll}
2.61 & 0.69 & 2.61 \\
0.69 & 2.61 & 2.61 \\
2.61 & 2.61 & 0.96
\end{array}\right), \\
& \mathbf{K}_{2}=\left(\begin{array}{lll}
3.65 & 0.98 & 3.65 \\
0.98 & 3.65 & 3.65 \\
3.65 & 3.65 & 1.34
\end{array}\right) .
\end{aligned}
$$

Next, the values continuously decrease toward initial values of $\mathbf{K}_{\mathbf{1}}$. Values of elements $\left(k_{i j}\right)$ of $\mathbf{K}_{\mathbf{1}}$ and $\mathbf{K}_{\mathbf{2}}$ are given in $10^{10} \mathrm{~Pa}$. Material 2 has the same parameters as material 1 , but elasticity tensor element $k_{13}=0$. Material 3 has element $k_{23}=0$. Material 4 corresponds to the transversely isotropic case, when $k_{13}=k_{23}=0$. Nonzero and zero elements of $\mathbf{K}$ for our materials are presented in Table 1.

Let us consider gradient anisotropic layer of thickness $L=10 \mathrm{~m}$ placed in sandstone. $P$-wave velocity of sandstone $v_{P}=3300 \mathrm{~m} / \mathrm{sec}$ and $S$-wave velocity $v_{S}=2000 \mathrm{~m} / \mathrm{sec}$. Density of all materials is $2400 \mathrm{~kg} / \mathrm{m}^{3}$. For experiments, we considered four cases of filling the gradient layer: by material 1 , by material 2 , by material 3 , and by material 4 .

Note that tensor $\mathbf{K}_{\mathbf{1}}$ for material 4 (i.e., when $k_{13}=k_{23}=$ 0 ) corresponds to sandstone enveloping the layer. Also, tensor $\mathbf{K}_{\mathbf{2}}$ also for material 4 corresponds to a harder sandstone with $v_{P}=3900 \mathrm{~m} / \mathrm{sec}$ and $v_{S}=2360 \mathrm{~m} / \mathrm{sec}$.

As a result of numerical experiments, the dependence of normalized energy flow on frequency for each material can be shown. The experiments show how various cases of anisotropy affect transmittance through gradient layers of complex structures.

While investigating the wave diffraction by layers, which are filled with material 1 (full anisotropy) and material 2 $\left(k_{13}=0\right)$, we made a conclusion that normalized energy flow of transmitted wave in the case of normal incidence for these materials leads to identical values. This is explained by the fact that elasticity tensor element $k_{13}$ is a proportionality coefficient, which shows how intensity of the rotational components of forces influences strain of the body along the $x$-axis. Since the longitudinal wave falling normally propagates along the $y$-axis, we set the element $k_{13}$ to a nonzero value and elasticity tensor $\mathbf{K}$ has no effect on wave propagation. The normalized energy flow of transmitted wave through material 3 coincides with that through material 4 .

Let us consider the dependence of normalized energy flow of transmitted longitudinal wave at the middle frequencies, when the layer is as thick as several wavelengths, and at the high frequencies, when the layer is as thick as tens 


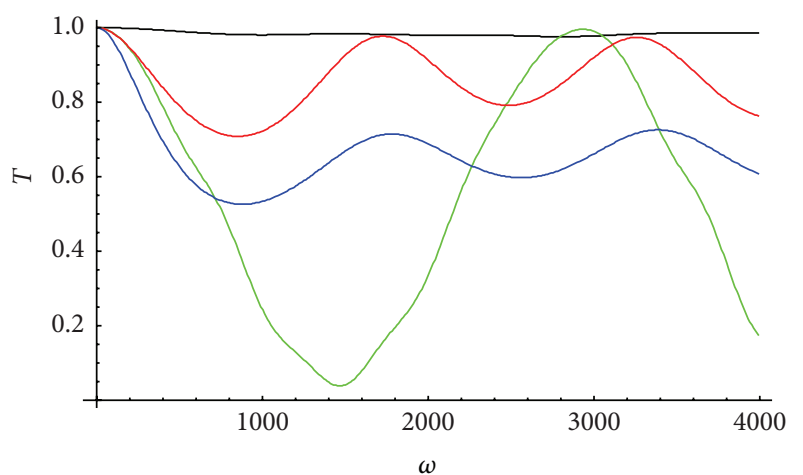

FIgURE 2: Transmittance of the layer placed in sandstone versus circular frequency $\omega$. Blue graph is for material 1. Red graph is for material 2. Green graph is for material 3. Black graph is for material 4. Thickness of the layer is $10 \mathrm{~m}$; incidence angle $\theta=30^{\circ}$.

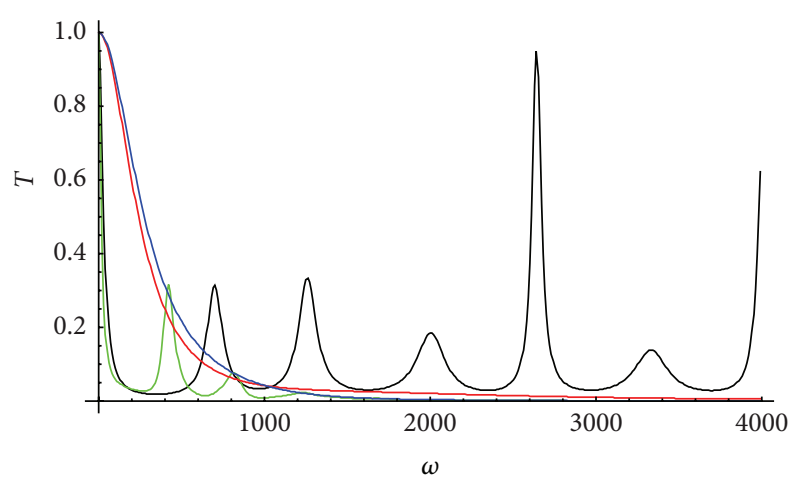

FIGURE 3: Transmittance of the layer placed in sandstone versus circular frequency $\omega$. Black graph is for material 1. Red graph is for material 2. Green graph is for material 3. Blue graph is for material 4. Thickness of the layer is $10 \mathrm{~m}$; incidence angle $\theta=85^{\circ}$.

of wavelength. Note that when the incidence angle is less than $45^{\circ}$, material 1 and material 2 show a similar behavior. Figure 2 shows dependence of the normalized transmitted energy flow on frequency $\omega$ at the incidence angle $\theta=30^{\circ}$. The figure shows that at the narrow incidence angles the shearing stress does not have a significant effect on energy flow of the transmitted wave, which means that the graph for material 1 shows the same behavior as the graph for material 2 . When the incidence angle is greater than $45^{\circ}$, such dependence is not observed.

Note that when the incidence angle is more than $70^{\circ}$ for the case of full anisotropy, at middle and high frequencies the band gaps (when we can see the transmitted energy jump) occur. Figure 3 shows dependence of the normalized energy flow of transmitted wave on frequency at the incidence angle of $85^{\circ}$. For material 1 at frequency near $710 \mathrm{rad} / \mathrm{sec}$ and $1280 \mathrm{rad} / \mathrm{sec}$, we can see the $30 \%$ transmitted energy gap, and at frequency $2720 \mathrm{rad} / \mathrm{sec}$ the gap becomes more than $90 \%$.

In Figure 3, the graph corresponding to the full anisotropy case has the band gap at frequency near $2720 \mathrm{rad} / \mathrm{sec}$. The

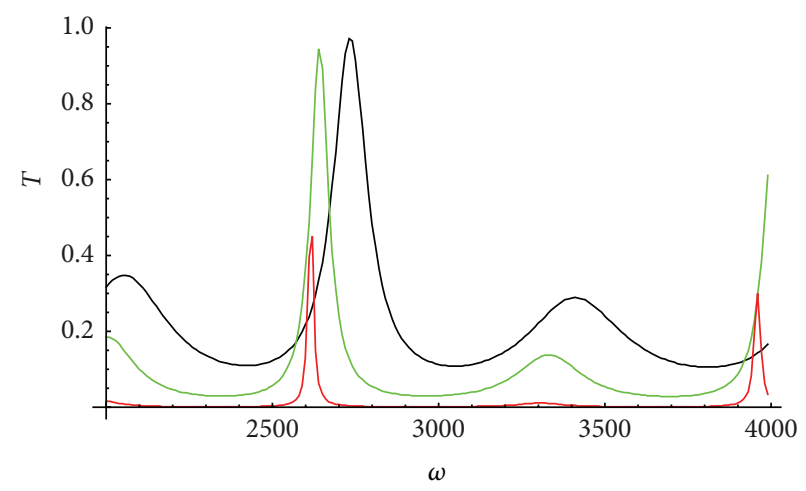

FIGURE 4: Transmittance of the layer filled with material 1 placed in sandstone versus circular frequency $\omega$. Red graph is for incident angle $\theta=89^{\circ}$. Green graph is for incident angle $\theta=85^{\circ}$. Black graph is for incident angle $\theta=80^{\circ}$. Thickness of the layer is $10 \mathrm{~m}$.

investigation of impact of incidence angle on characteristics of transmitted energy gap results in the following conclusions: the width of the frequency window becomes narrower when the incidence angle increases. The fact is confirmed by graphs of Figure 4, where the dependence of normalized energy flow of transmitted wave on frequency at different angles is presented.

We can see the distinctively expressed band gaps for material 1 (with full anisotropy) at the angle interval from $80^{\circ}$ to $89^{\circ}$.

The gradient layer considered above is a synthetic layer. Let us stop at one example, in which the parameters of the layer at the boundary and in the center correspond to the real rocks and vary in the continuous manner, as in the first example. Let these substances be amazonite (tensor $\mathbf{K}_{1}$ ) at the layer's boundaries and labradorite (tensor $\mathbf{K}_{2}$ ) in the middle [22]:

$$
\begin{aligned}
& \mathbf{K}_{\mathbf{1}}=\left(\begin{array}{ccc}
5.7 & 3.36 & -1.26 \\
3.36 & 10.2 & -1.14 \\
-1.26 & -1.14 & 2.09
\end{array}\right), \\
& \mathbf{K}_{2}=\left(\begin{array}{ccc}
98.8 & 43.7 & -10.2 \\
43.7 & 141.0 & -18.0 \\
-10.2 & -18.0 & 34.3
\end{array}\right),
\end{aligned}
$$

where values $k_{i j}$ are determined in $10^{10} \mathrm{~Pa}$. Note that the substances filling the layer are feldspars.

Results of calculations of energy transmitted through the layer are given in Figure 5. The black line corresponds to the case of normal incidence onto the layer; the green line corresponds to the incidence angle $\theta=30^{\circ}$; the red line is for $\theta=60^{\circ}$; the blue line is for $\theta=80^{\circ}$.

\section{Conclusions}

The method of the overdetermined boundary value problem, used in the present work, when combined with the Fourier transform method, is shown to be efficient, especially for the 


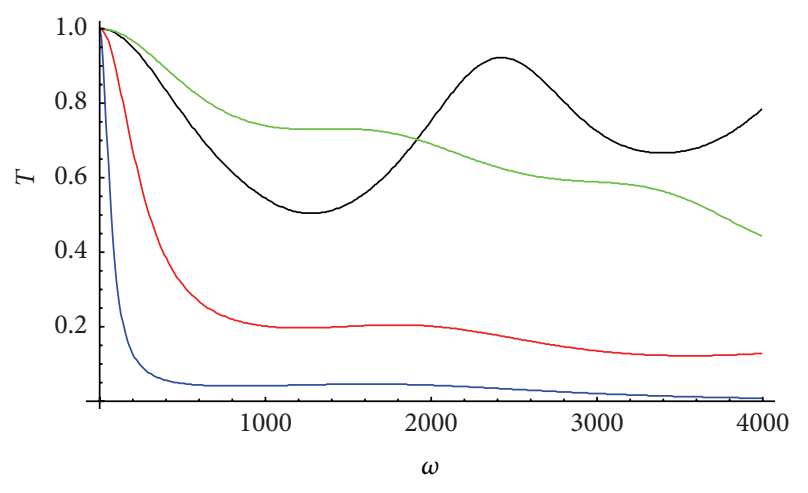

FIGURE 5: Transmittance of the layer versus circular frequency $\omega$. Medium of the layer is continuously changing from amazonite to labradorite. The layer is placed in sandstone. Black graph corresponds to normal incidence, green graph is for incident angle $\theta=$ $30^{\circ}$, red graph is for $\theta=60^{\circ}$, and blue graph is for $\theta=80^{\circ}$. Thickness of the layer is $10 \mathrm{~m}$.

cases in which the Fourier transforms of traces of the incident field are singular distributions. Then the approximation problem is solved just at the value $\xi=\xi_{0}$. In the case of the Fourier transforms being regular distributions (e.g., at diffraction of a Gauss beam by a plate), problem (7), (8), and (11) is solved for several values of the parameter $\xi$.

In the case of normal incidence of the wave onto the layer, the normalized energy flow of transmitted wave for the fully anisotropic material coincides with that for material with $k_{13}=0$; for the transversely isotropic material and material with element $k_{23}=0$, a similar coincidence is found.

When the incident angle is less than $45^{\circ}$, the shearing stress has a small effect on energy flow of transmitted wave. Consequently, the values of elasticity tensor element $k_{13}$ do not influence the diffraction process.

For the big incidence angles range (more than $85^{\circ}$ ) of materials with full anisotropy, the energy flow of the transmitted wave at the middle and high frequencies can have bands, in which all energy passes through the layer. Width of the frequency interval becomes narrower with increasing the incidence angle.

The results obtained for elastic wave transmittance through gradient-type anisotropic layers can be used in geophysics for primary analysis of the geological formation structure. Also, the results on elastic wave transmittance through nonuniform anisotropic structures can be used in industries, where anisotropic materials are applied, for designing protective layers of various structures.

\section{Disclosure}

The work is performed according to the Russian Government Program of Competitive Growth of Kazan Federal University.

\section{Conflict of Interests}

The authors declare that there is no conflict of interests regarding the publication of this paper.

\section{References}

[1] V. Babuska and M. Cara, Seismic Anisotropy in the Earth, Springer Science \& Business Media, 1991.

[2] C. M. Sayers and M. Kachanov, "Microcrack-induced elastic wave anisotropy of brittle rocks," Journal of Geophysical Research, vol. 100, no. 3, pp. 4149-4156, 1995.

[3] R. T. Bachman, "Elastic anisotropy in marine sedimentary rocks," Journal of Geophysical Research: Solid Earth, vol. 88, no. 1, pp. 539-545, 1983.

[4] M. Schoenberg and C. M. Sayers, "Seismic anisotropy of fractured rock," Geophysics, vol. 60, no. 1, pp. 204-211, 1995.

[5] I. Tsvankin, J. Gaiser, V. Grechka, M. Van der Baan, and L. Thomsen, "Seismic anisotropy in exploration and reservoir characterization: an overview," Geophysics, vol. 75, no. 5, pp. 75A15-75A29, 2010.

[6] P. G. Silver, "Seismic anisotropy beneath the continents: probing the depths of geology," Annual Review of Earth and Planetary Sciences, vol. 24, pp. 385-432, 1996.

[7] S.-I. Karato, H. Jung, I. Katayama, and P. Skemer, "Geodynamic significance of seismic anisotropy of the upper mantle: new insights from laboratory studies," Annual Review of Earth and Planetary Sciences, vol. 36, pp. 59-95, 2008.

[8] N. N. Puzyrev, I. R. Obolentseva, A. V. Trigubov, and S. B. Gorshkalev, "On the anisotropy of sedimentary rocks from shear-wave analysis," Geophysical Journal International, vol. 76, no. 1, pp. 243-252, 1984.

[9] M. S. Savage, "Seismic anisotropy and mantle deformation: what have we learned from shear wave splitting?" Reviews of Geophysics, vol. 37, no. 1, pp. 65-106, 1999.

[10] J. Li, Z. Liang, J. Zhu, and X. Zhangh, Acoustic Metamaterials: Negative Refraction, Imaging, Lensing and Cloaking, vol. 166, Springer Science \& Business Media, 2012.

[11] R. Zhu, X. N. Liu, G. L. Huang, H. H. Huang, and C. T. Sun, "Microstructural design and experimental validation of elastic metamaterial plates with anisotropic mass density," Physical Review B, vol. 86, no. 14, Article ID 144307, 2012.

[12] A. P. Liu, R. Zhu, X. N. Liu, G. K. Hu, and G. L. Huang, "Multi-displacement microstructure continuum modeling of anisotropic elastic metamaterials," Wave Motion, vol. 49, no. 3, pp. 411-426, 2012.

[13] D. Torrent and J. Sánchez-Dehesa, "Acoustic cloaking in two dimensions: a feasible approach," New Journal of Physics, vol. 10, no. 6, Article ID 063015, 2008.

[14] D. L. Anderson, "Elastic wave propagation in layered anisotropic media," Journal of Geophysical Research, vol. 66, no. 9, pp. 2953-2963, 1961.

[15] A. V. Anufrieva, D. N. Tumakov, and V. L. Kipot, "Peculiarities of propagation of a plane elastic wave through a gradient layer," in Proceedings of the Days on Diffraction (DD '13), pp. 11-16, IEEE, Saint Petersburg, Russia, May 2013.

[16] A. V. Anufrieva and D. N. Tumakov, "Diffraction of a plane elastic wave by a gradient transversely isotropic layer," Advances in Acoustics and Vibration, vol. 2013, Article ID 262067, 8 pages, 2013.

[17] A. V. Anufrieva and D. N. Tumakov, "On some of the peculiarities of propagation of an elastic wave through a gradient transversely isotropic layer," in Proceedings of the Days on Diffraction (DD '14), pp. 23-28, IEEE, Saint Petersburg, Russia, May 2014. 
[18] L. A. Tolokonnikov, "Reflection and refraction of a planar acoustic wave in an anisotropic inhomogeneous layer," Journal of Applied Mechanics and Technical Physics, vol. 40, no. 5, pp. 936-941, 1999.

[19] L. A. Tolokonnikov, "The transmission of sound through an inhomogeneous anisotropic layer adjoining viscous liquids," Journal of Applied Mathematics and Mechanics, vol. 62, no. 6, pp. 953-958, 1998.

[20] I. E. Pleshchinskaya and N. B. Pleshchinskii, "Over-determined boundary value problems for linear equations of elastodynamics and their applications to elastic wave diffraction theory," Advances in Mathematics Research, vol. 17, pp. 102-138, 2012.

[21] A. V. Anufrieva, K. B. Igudesman, and D. N. Tumakov, "Peculiarities of elastic wave refraction from the layer with fractal distribution of density," Applied Mathematical Sciences, vol. 8, no. 117-120, pp. 5875-5886, 2014.

[22] A. G. Every and A. K. McCurdy, Second and Higher Order Elastic Constants, vol. III/29A of Landolt-Börnstein, Springer, Berlin, Germany, 1992. 

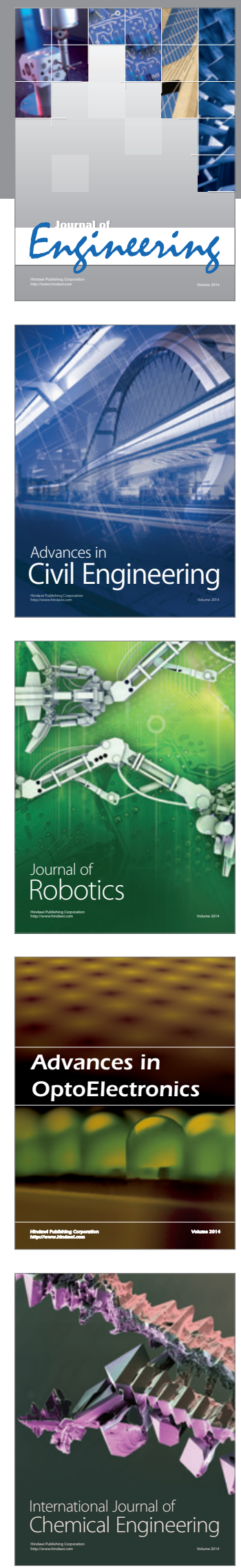

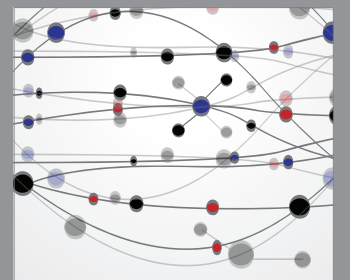

The Scientific World Journal
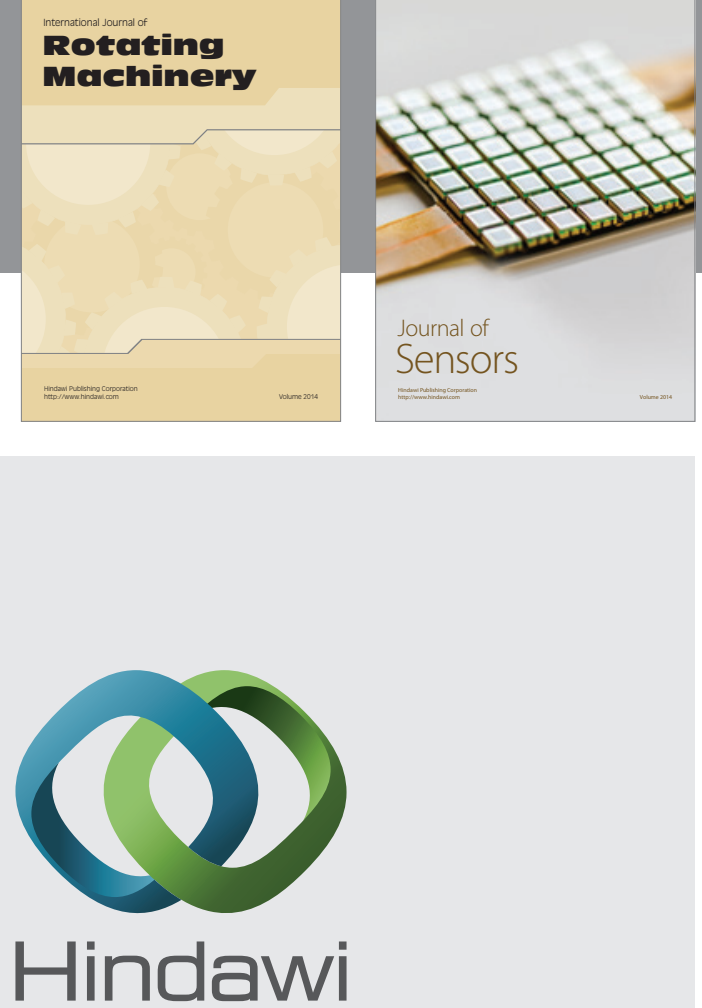

Submit your manuscripts at http://www.hindawi.com
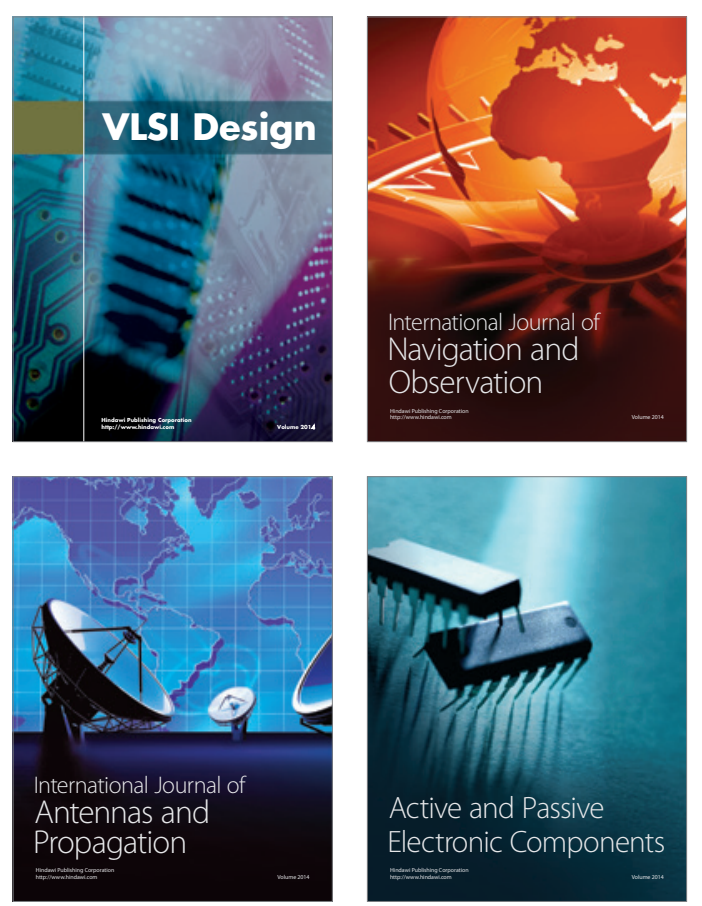
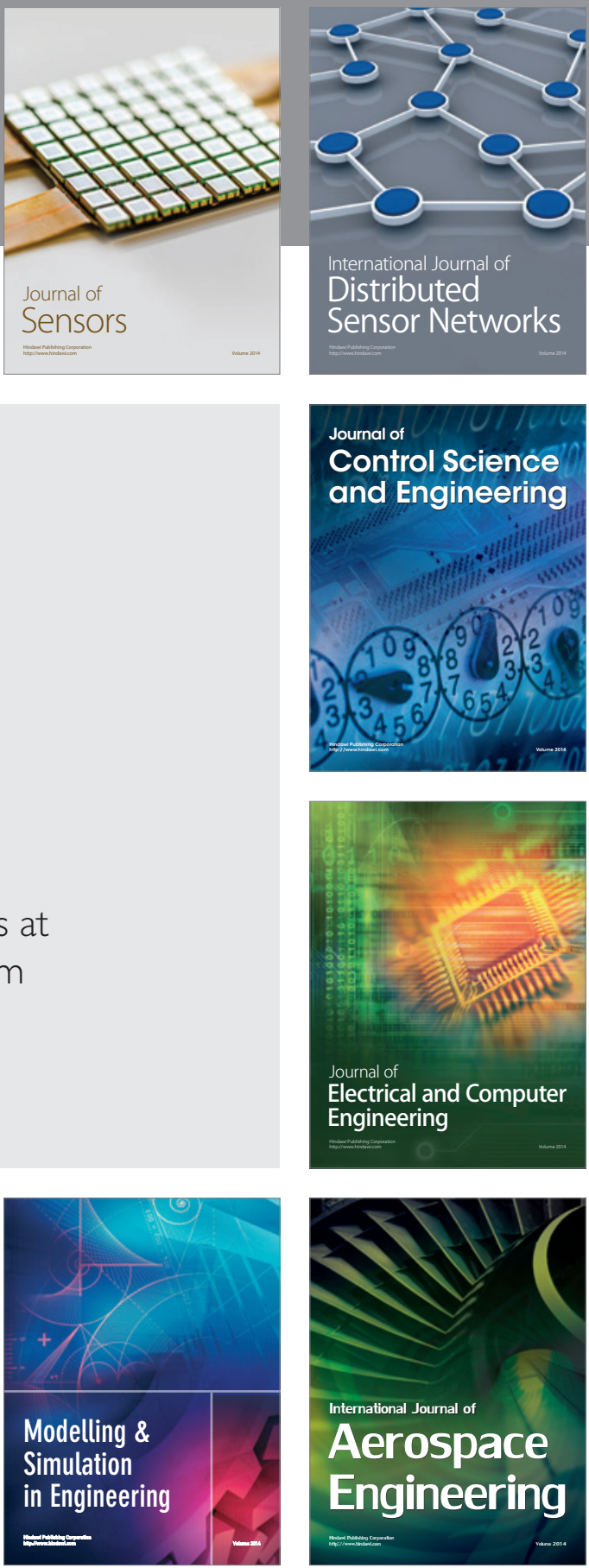

Journal of

Control Science

and Engineering
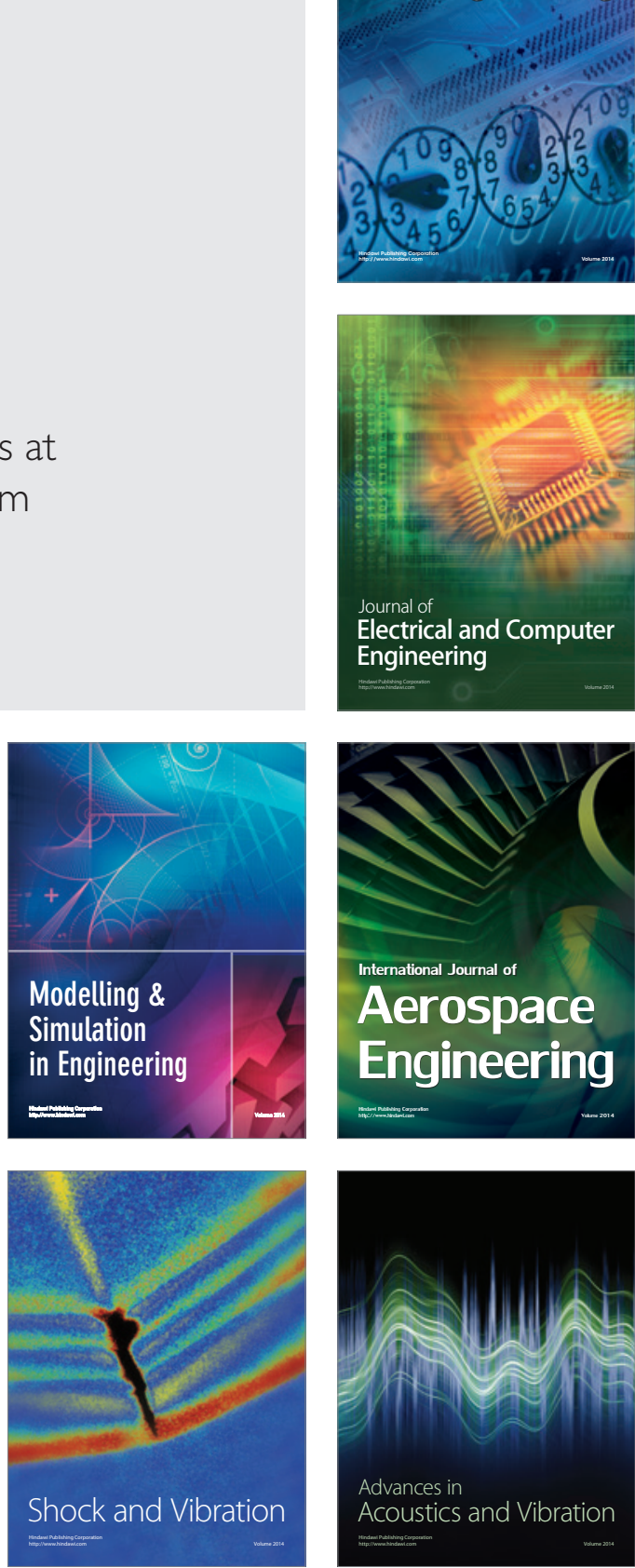